\title{
Cyclic polygons in non-Euclidean geometry
}

Lienhard Wimmer

Lienhard Wimmer studierte Mathematik an der Paris-Lodron-Universität in Salzburg. Nach einem Studienaufenthalt in Marseille promovierte er in Salzburg über die gleichmäßige Verteilung von Punkten auf der Einheitskugel. Er arbeitet als Softwareentwickler in München.

It is well-known that in Euclidean geometry among all quadrilaterals with prescribed edges the cyclic quadrilateral, i.e. the quadrilateral whose vertices all belong to a single circle, has largest area. In fact this theorem is valid for every polygon. But does it also hold in non-Euclidean geometry?

In this note we answer this question for hyperbolic and spherical polygons. Of course we are only interested in non-degenerate triangles. Therefore in the hyperbolic plane $H^{2}$ we study only polygons consisting of non-asymptotic triangles, i.e. triangles with vertices belonging to the finite part of the plane, and on the sphere $S^{2}$ we study only polygons which do not exceed the half-sphere and consist of Eulerian triangles, i.e. triangles whose angles and edges are $<\pi$.

\section{Area of a non-Euclidean triangle}

A good method to handle plane non-Euclidean geometry is the use of trigonometry. It is therefore appropriate to point out some elementary formulas concerning triangles in hyperbolic and spherical geometry.

\subsection{Hyperbolic geometry}

We start with the trigonometry of the hyperbolic plane. Let $\triangle A B C$ be a non-asymptotic triangle, let $a:=B C, b:=C A$, and $c:=A B$ be its edges and $\alpha:=\angle C A B, \beta:=\angle A B C$,

Ein Sehnenviereck ist ein Viereck, dessen Eckpunkte auf einem Kreis liegen. Bekanntlich besitzt unter allen Vierecken mit gegebenen Seiten das Sehnenviereck die größte Fläche. Doch gilt diese Aussage auch in der nicht-euklidischen Geometrie? Im vorliegenen Beitrag beantwortet der Autor diese Frage vollständig für Vierecke der hyperbolischen und der sphärischen Geometrie. 
and $\gamma:=\angle B C A$ be its angles. Then, the theorem of sine for the non-asymptotic triangle $\triangle A B C$ is given by

$$
\frac{\sin \alpha}{\sinh a}=\frac{\sin \beta}{\sinh b}=\frac{\sin \gamma}{\sinh c},
$$

and there are two theorems of cosine

$$
\begin{aligned}
\cosh c & =\cosh a \cdot \cosh b-\sinh a \cdot \sinh b \cdot \cos \gamma, \\
\cos \gamma & =-\cos \alpha \cdot \cos \beta+\sin \alpha \cdot \sin \beta \cdot \cosh c .
\end{aligned}
$$

Lemma 1. In hyperbolic geometry the edges and angles of a non-asymptotic triangle fulfil the following equations

$$
\begin{aligned}
\sinh a \cdot \sinh b \cdot \sin \gamma & =2 \cdot \cosh ^{2} \frac{c}{2} \cdot \frac{\cos \gamma+\cos (\alpha+\beta)}{\sin \gamma}, \\
\tan \frac{\alpha+\beta}{2} & =\frac{\cosh \frac{a-b}{2}}{\cosh \frac{a+b}{2}} \cdot \cot \frac{\gamma}{2}
\end{aligned}
$$

Proof. We start with the addition theorems of the hyperbolic functions

$$
\cosh (x \pm y)=\cosh x \cdot \cosh y \pm \sinh x \cdot \sinh y .
$$

From these equations we get

$$
\begin{aligned}
\cosh x+\cosh y & =2 \cdot \cosh \frac{x+y}{2} \cdot \cosh \frac{x-y}{2}, \\
\cosh (2 x) & =\sinh ^{2} x+\cosh ^{2} x=1+2 \cdot \sinh ^{2} x,
\end{aligned}
$$

together with the two half-angle-relations

$$
\begin{aligned}
& \cosh x \cdot \cosh y=\cosh ^{2} \frac{x+y}{2}+\cosh ^{2} \frac{x-y}{2}-1, \\
& \sinh x \cdot \sinh y=\cosh ^{2} \frac{x+y}{2}-\cosh ^{2} \frac{x-y}{2} .
\end{aligned}
$$

For a non-degenerate triangle in the hyperbolic plane we get immediately from the second theorem of cosine

$$
\frac{\cos \gamma+\cos \alpha \cdot \cos \beta}{\sin \alpha \cdot \sin \beta}=\cosh c=1+2 \cdot \sinh ^{2} \frac{c}{2},
$$

resp.

$$
2 \cdot \sinh ^{2} \frac{c}{2}=\frac{\cos \gamma+\cos (\alpha+\beta)}{\sin \alpha \cdot \sin \beta} .
$$

With the theorem of sine this gives the first equation of the lemma because of

$$
\sinh a \cdot \sinh b \cdot \sin \gamma=\frac{\sin \alpha \cdot \sin \beta}{\sin \gamma} \cdot \sinh ^{2} c .
$$


For the proof of the second equation we need

$$
(\cosh a+\cosh b) \cdot \sin \gamma=\sin (\alpha+\beta) \cdot(1+\cosh c) .
$$

We get this equation from $\sin (\alpha+\beta)=\sin \alpha \cdot \cos \beta+\cos \alpha \cdot \sin \beta$, if we replace $\sin \alpha$ and $\cos \alpha$, resp. $\sin \beta$ and $\cos \beta$ by the terms we get from the theorem of sine and the first theorem of cosine.

From (3) and the second law of cosine we get

$$
\sin (\alpha+\beta)=\frac{(\cosh a+\cosh b) \cdot \sin \gamma}{1+\cosh a \cdot \cosh b-\sinh a \cdot \sinh b \cdot \cos \gamma}
$$

By the half-angle-relations this is equal to

$$
\sin (\alpha+\beta)=\frac{2 \cdot \cosh \frac{a+b}{2} \cdot \cosh \frac{a-b}{2} \cdot \sin \gamma}{\cosh ^{2} \frac{a+b}{2} \cdot(1-\cos \gamma)+\cosh ^{2} \frac{a-b}{2} \cdot(1+\cos \gamma)},
$$

resp.

$$
\frac{2 \cdot \tan \frac{\alpha+\beta}{2}}{1+\tan ^{2} \frac{\alpha+\beta}{2}}=\sin (\alpha+\beta)=\frac{2 \cdot \frac{\cosh \frac{a-b}{2}}{\cosh \frac{a+b}{2}} \cdot \cot \frac{\gamma}{2}}{1+\frac{\cosh ^{2} \frac{a-b}{2}}{\cosh ^{2} \frac{a+b}{2}} \cdot \cot ^{2} \frac{\gamma}{2}}
$$

the second equation of the lemma follows directly.

\subsection{Spherical geometry}

In spherical geometry we use the same notations for the edges and angles of an Eulerian triangle. By similar considerations as in the hyperbolic case we get

$$
\begin{aligned}
-\sin a \cdot \sin b \cdot \sin \gamma & =2 \cdot \cos ^{2} \frac{c}{2} \cdot \frac{\cos \gamma+\cos (\alpha+\beta)}{\sin \gamma}, \\
\tan \frac{\alpha+\beta}{2} & =\frac{\cos \frac{a-b}{2}}{\cos \frac{a+b}{2}} \cdot \cot \frac{\gamma}{2}
\end{aligned}
$$

\section{Quadrilaterals with maximal area}

Let \#ABCD be a quadrilateral in non-Euclidean geometry, let its edges be given by $a:=$ $A B, b:=B C, c:=C D$, and $d:=D A$, and let $t$ be the diagonal $B D$. The angles of the quadrilateral shall be given by $\alpha:=\angle D A B, \beta:=\angle A B C, \gamma:=\angle B C D$, and $\delta:=\angle C D A$; furthermore let $\varphi:=\angle A B D+\angle B D A$ and $\psi:=\angle C D B+\angle C B D$.

Theorem 1. Among all non-Euclidean quadrilaterals with given sides there is a quadrilateral with largest area; it is characterized by $\alpha+\gamma=\beta+\delta$. 


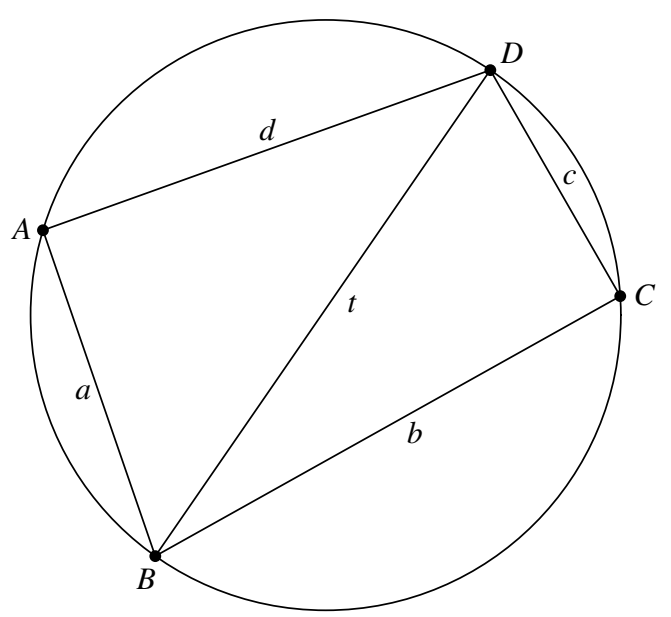

Fig. 1 Cyclic quadrilateral

Proof. The area $\epsilon$ of the quadrilateral is given by the sum or the difference of the area of the triangles $\triangle A B D$ and $\triangle B C D$ (see Fig. 1); of course the largest area can only be given by the sums. We consider $\epsilon$ to be a continuous function of $t$. The smallest possible value of $t$ is given by the maximum of $a-b$ and $c-d$. Starting at this value and increasing $t$ the area obviously also increases. In converse, the area decreases if we start at an appropriate value of $t$ near the maximal possible value (i.e. the minimum of $a+b$ and $c+d$ ) and decrease $t$. Between the smallest and largest possible values for $t$ the area of the quadrilateral is continuous as a function of $t$. It attains its maximum for a value $t_{0}$ between the minimal and maximal possible value; at $t_{0}$ the first derivative necessarily has to vanish.

It is easy to express the area $\epsilon$ of the quadrilateral by its angles, because the area of $\triangle A B D$ is given by

$$
\epsilon_{1}:= \begin{cases}(\alpha+\varphi)-\pi & \triangle A B D \subset S^{2}, \\ \pi-(\alpha+\varphi) & \triangle A B D \subset H^{2},\end{cases}
$$

and the area of $\triangle B C D$ is given by

$$
\epsilon_{2}:= \begin{cases}(\gamma+\psi)-\pi & \triangle B C D \subset S^{2}, \\ \pi-(\gamma+\psi) & \triangle B C D \subset H^{2} .\end{cases}
$$

Thus $\epsilon=\epsilon_{1}+\epsilon_{2}$ depends linearily on

$$
p=\left(\alpha+2 \cdot \arctan \left(K_{a d} \cdot \cot \frac{\alpha}{2}\right)\right)+\left(\gamma+2 \cdot \arctan \left(K_{b c} \cdot \cot \frac{\gamma}{2}\right)\right)
$$

with constants $K_{a d}$ and $K_{b c}$ only depending on $a$ and $d$, resp. $b$ and $c$. This follows immediately from (5), resp. (2).

Our aim is to find the extremal value of $p$, resp. $\epsilon$ under the condition

$$
\cos a \cdot \cos d+\sin a \cdot \sin d \cdot \cos \alpha=\cos B D=\cos b \cdot \cos c+\sin b \cdot \sin c \cdot \cos \gamma .
$$


We assume that $\alpha$ and $\gamma$ depend strictly monotone on $t$. Then we get

$$
\begin{aligned}
& \frac{d}{d t} \epsilon_{1}=\frac{d}{d t}\left(\alpha+2 \cdot \arctan \left(K_{a d} \cdot \cot \frac{\alpha}{2}\right)\right)=\left(1-\frac{K_{a d} \cdot \csc ^{2} \frac{\alpha}{2}}{1+K_{a d}^{2} \cdot \cot ^{2} \frac{\alpha}{2}}\right) \cdot \frac{d \alpha}{d t}, \\
& \frac{d}{d t} \epsilon_{2}=\frac{d}{d t}\left(\gamma+2 \cdot \arctan \left(K_{b c} \cdot \cot \frac{\gamma}{2}\right)\right)=\left(1-\frac{K_{b c} \cdot \csc ^{2} \frac{\gamma}{2}}{1+K_{b c}^{2} \cdot \cot ^{2} \frac{\gamma}{2}}\right) \cdot \frac{d \gamma}{d t} .
\end{aligned}
$$

Reexpressing $K_{a d}$ by $\tan \frac{\varphi}{2} \cdot \tan \frac{\alpha}{2}$ and $K_{b c}$ by $\tan \frac{\psi}{2} \cdot \tan \frac{\gamma}{2}$, we find for the first derivative of $p$, resp. $\epsilon$

$$
\frac{d p}{d t}=\left(1-\frac{\sin \varphi}{\sin \alpha}\right) \cdot \frac{d \alpha}{d t}+\left(1-\frac{\sin \psi}{\sin \gamma}\right) \cdot \frac{d \gamma}{d t} .
$$

From condition (6) we get

$$
\sin a \cdot \sin d \cdot \sin \alpha \cdot \frac{d \alpha}{d t}=\sin b \cdot \sin c \cdot \sin \gamma \cdot \frac{d \gamma}{d t} .
$$

Because of (4), resp. (1) this is equal to

$$
\left(\frac{\cos \alpha+\cos \varphi}{\sin \alpha}\right) \cdot \frac{d \alpha}{d t}-\left(\frac{\cos \gamma+\cos \psi}{\sin \gamma}\right) \cdot \frac{d \gamma}{d t}=0 .
$$

Therefore the first derivative of $p$, resp. $\epsilon$ can only vanish under the given condition, if

$$
\frac{\sin \alpha-\sin \varphi}{\cos \alpha+\cos \varphi}+\frac{\sin \gamma-\sin \psi}{\cos \gamma+\cos \psi}=0
$$

resp.

Thus

$$
\frac{2 \cdot \cos \frac{\alpha+\varphi}{2} \cdot \sin \frac{\alpha-\varphi}{2}}{2 \cdot \cos \frac{\alpha+\varphi}{2} \cdot \cos \frac{\alpha-\varphi}{2}}=-\frac{2 \cdot \cos \frac{\gamma+\psi}{2} \cdot \sin \frac{\gamma-\psi}{2}}{2 \cdot \cos \frac{\gamma+\psi}{2} \cdot \cos \frac{\gamma-\psi}{2}}
$$

resp.

$$
\tan \frac{\alpha-\varphi}{2}=-\tan \frac{\gamma-\psi}{2}=\tan \frac{\psi-\gamma}{2}
$$

$$
\alpha+\gamma=\varphi+\psi=\beta+\delta
$$

\section{Non-Euclidean cyclic quadrilaterals}

Among all quadrilaterals with edges of given length the quadrilateral with largest area necessarily fulfils the condition of Theorem 1 . This condition is valid for all cyclic quadrilaterals.

Lemma 2. The angles of a cyclic quadrilateral \#ABCD fulfil the equation

$$
\alpha+\gamma=\beta+\delta
$$

Proof. Let $M$ be the center of the circumcircle of the quadrilateral. Then the triangles $\triangle M A B, \triangle M B C, \triangle M C D$, and $\triangle M D A$ are isosceles and the angles at $A$ and $B$ etc. are equal. The equation follows immediately. 
But does the quadrilateral with the largest area always have to be cyclic? In Euclidean and spherical geometry the answer is "yes", but in hyperbolic geometry the answer cannot be as clear as that, because even triangles do not necessarily have a circumcircle there.

Lemma 3. In Euclidean and spherical geometry a convex quadrilateral with $\alpha+\gamma=\beta+\delta$ is cyclic.

Proof. In Euclidean geometry the angles of a quadrilateral \#ABCD sum up to $2 \pi$; from the assumed equality we get therefore $\beta+\delta=\pi$ and $\alpha+\gamma=\pi$. Consider the circumcircle $k$ of $\triangle A B C$ and consider $A C$ to be a chord of it. The vertex $D$ cannot belong to the same side of $A C$ as $B$, because the quadrilateral would not be convex in that case. By the converse of the Common Chord Theorem and because of $\delta=\pi-\beta$ we find that the vertex $D$ has to be a part of the second arc of $k$.

In spherical geometry two great circles always intersect. Therefore we can use the following construction:

Case 1: $\alpha \neq \beta$. Without loss of generality we may assume that $\alpha>\delta$ and $\beta>\gamma$. We construct the line $f$ passing through $A$ with $\angle D A P=\delta$ for each point $P \in f$ and the line $g$ passing through $B$ with $\angle Q B C=\gamma$ for each point $Q \in g$. Let $h$ be the great circle defined by $C$ and $D$. Then let $C^{\prime}$ be the intersection of $g$ and $h$, let $D^{\prime}$ be the intersection of $f$ and $h$, and let $E^{\prime}$ be the intersection of $f$ and $g$ (see Fig. 2).

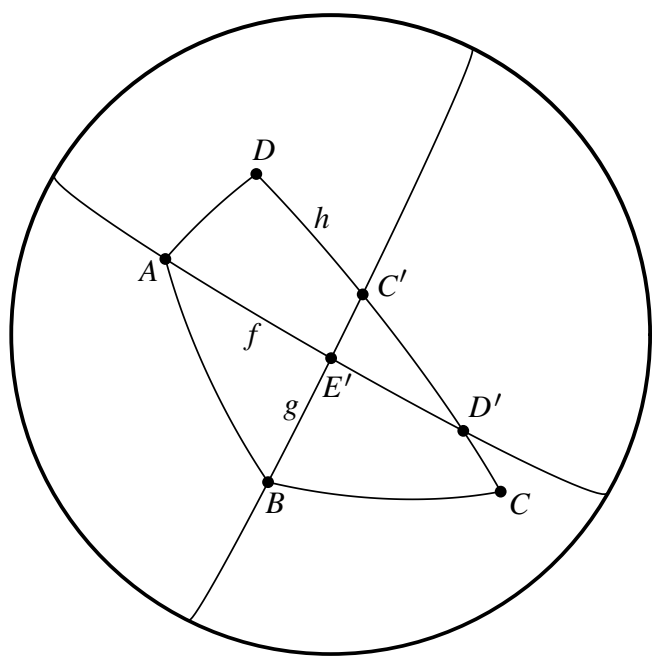

Fig. 2 Spherical quadrilateral

$\triangle A D^{\prime} D$ and $\triangle C C^{\prime} B$ are isosceles triangles because they have the same angle at the bottom line $A D$, resp. $B C$. Thus the angle bisector at $D^{\prime}$, resp. $C^{\prime}$ is the median line of $A D$, resp. $B C$.

Let us study the triangle $\triangle A B E^{\prime}$ : its angle at $A$ is given by $\alpha-\delta$ and its angle at $B$ is given by $\beta-\gamma$. By assumption these angles are equal and $\triangle A B E^{\prime}$ is therefore also an isosceles triangle; thus the angle bisector at $E^{\prime}$ is the median line of $A B$. 
The points $C^{\prime}, D^{\prime}$, and $E^{\prime}$ form a triangle. The bisectors of the angles of a triangle intersect at the incenter of $\triangle C^{\prime} D^{\prime} E^{\prime}$, which we name $U$. The distance from $U$ to $B$ and $C$ is equal because the bisector of the triangle at $C^{\prime}$ is - by construction - the median line of $B C$. By the same argument we get that $U$ has equal distance from $A, B, C$, and $D$; i.e. \#ABCD is cyclic with center $U$.

Case 2: $\alpha=\beta$, i.e. $\gamma=\delta$. Then the quadrilateral is a convex isosceles trapezoid and therefore the median line of $A D$ is also the median line of $B C$. Let $U$ be the intersection point of $A B$ and $A D$; then $U$ is equidistant to $A, B, C$, and $D$.

To understand the hyperbolic geometry we use the Poincaré disc model. In this model the hyperbolic plane is given by the interior of a circle $\mathcal{C}$, hyperbolic lines are represented by arcs of circles that are orthogonal to $\mathcal{C}$ plus diameters of $\mathcal{C}$. This model has three important properties: angles in the model and angles in the hyperbolic plane are equal, a circle in the hyperbolic plane is represented by a Euclidean circle, and circumcircles of the Euclidean triangles are also circumcircles of their hyperbolic counterparts.

All Euclidean triangles have a circumcircle. In the hyperbolic context this circle is a

circumcircle if it is completely contained in the interior of $\mathcal{C}$,

horocycle if it touches the boundary of $\mathcal{C}$,

hypercycle if it intersects the boundary of $\mathcal{C}$.

Lemma 4. In hyperbolic geometry a convex quadrilateral \#ABCD with $\alpha+\gamma=\beta+\delta$ is inscribed into a circle, a horocycle, or a hypercycle.

Proof. We use the Poincaré disc model with boundary circle $\mathcal{C}$. Within this model we draw the quadrilateral \#ABCD. Then we may consider the drawing as Euclidean and add the Euclidean lines joining the vertices $A$ and $B, B$ and $C, C$ and $D$, resp. $D$ and $A$ (s. Fig. 3). In the Euclidean context hyperbolic lines are Euclidean circles intersecting orthogonally the boundary of $\mathcal{C}$. Thus the Euclidean angles $p$ and $p^{\prime}$ at $A$ and $B$ defined by the hyperbolic line $A B$ and their Euclidean counterpart are equal. The same is valid for the other lines. If we name these angles between the Euclidean and the hyperbolic lines with $p=p^{\prime}, q=q^{\prime}, r=r^{\prime}$, and $s=s^{\prime}$ we get for the Euclidean angles at $A, B, C$, and $D$

$$
\begin{aligned}
& \alpha_{E}=s^{\prime}+\alpha+p=s+\alpha+p, \\
& \beta_{E}=p^{\prime}+\beta+q=p+\beta+q, \\
& \gamma_{E}=q^{\prime}+\gamma+r=q+\gamma+r, \\
& \delta_{E}=r^{\prime}+\delta+s=r+\delta+s,
\end{aligned}
$$

resp.

$$
\alpha_{E}+\gamma_{E}=s+\alpha+p+q+\gamma+r=p+\beta+q+r+\delta+s=\beta_{E}+\delta_{E} .
$$

Therefore the vertices form a cyclic quadrilateral in Euclidean geometry, i.e. there is an Euclidean circle $k$ joining the vertices. This circle also passes through the vertices $A, B$, 


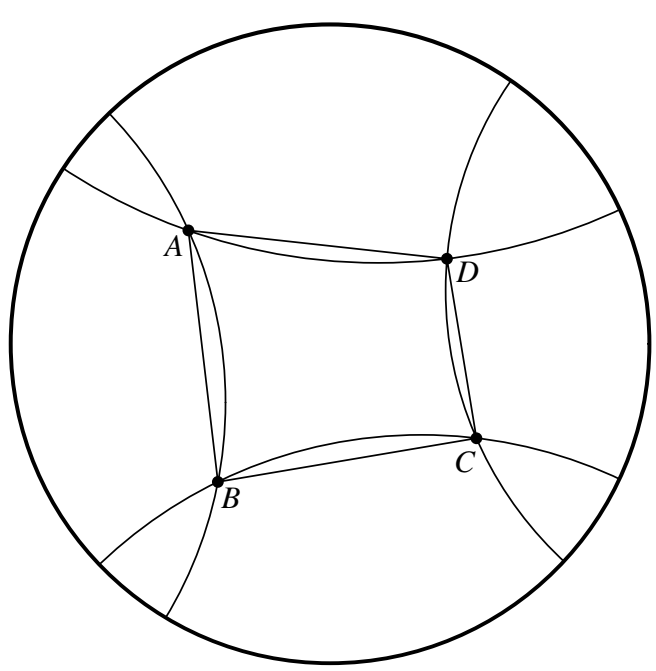

Fig. 3 Hyperbolic quadrilateral

$C, D$ if we regard them as points of the hyperbolic plane. The actual meaning of $k$ in the hyperbolic context, i.e. if it is a circle, a horocycle, or a hypercycle, depends on its intersection with the boundary of $\mathcal{C}$ (see the notes above concerning the circumcircle of the hyperbolic triangle).

\section{Applications to spherical geometry}

\subsection{Cyclic polygons}

In Euclidean and spherical geometry we can summarize our results as follows.

Proposition 1. In Euclidean and spherical geometry among all quadrilaterals with given sides the cyclic quadrilateral has largest area.

Following the proof of van der Waerden [3] it is easy to extend this theorem to polygons with $n$ given sides.

Theorem 2. In spherical geometry among all polygons with $n$ given sides, contained in a half-sphere, the polygon inscribed into a circle has largest area.

Proof (see [3]). From a vertex we draw the diagonals $x, y, \ldots$ etc. (see Fig. 4). Then we get $n-2$ triangles $a, b, x, x, c, y$, etc. The area of the polygon is the sum of the areas of these triangles. Of course some of them may have negative area, but it is clear that for given $x, y, \ldots$ the area is maximal if the area of all triangles is positive. The area is thus the sum of a continuous function depending on $x, y, \ldots$ These parameters are taken from a closed region defined by the triangle inequalities

$$
a+b \geq x, \quad x+y \geq c, \quad \text { etc. }
$$

The function therefore has a maximum. 


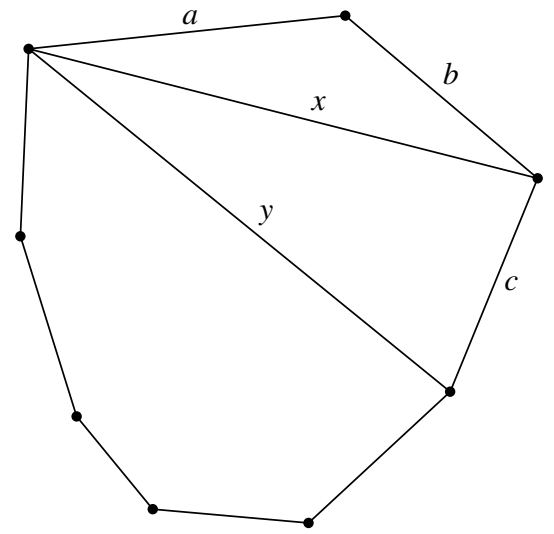

Fig. 4 Cyclic polygon

What does this maximum look like? If we only change the parameter $x$ we get from the already proven result that the vertices $A, B, C$, and $D$ are on the boundary of a circle. By the same argument we get that $B, C, D, E$ are also on the boundary of a circle. The vertices $B, C$, and $D$ belong to both circles and thus these circles are equal, i.e. the vertices $A, B, C, D$, and $E$ belong to the boundary of the same circle. If we apply this argument to all other vertices of the maximal polygon we get that the maximal polygon has to be cyclic.

\subsection{Tangential polygons}

The polar counterpart of a cyclic polygon is the tangential polygon, i.e. a polygon which is circumscribed an incircle. If $a, b, c$, and $d$ denote the sides of the quadrilateral, it is characterised by

$$
a+c=b+d
$$

and without any further proof we get from the duality of spherical geometry:

Theorem 3. Among all polygons with $n$ given angles the polygon circumscribing a circle has smallest area.

\section{References}

[1] Royster, D.: Non-Euclidean Geometry. http: //education. uncc. edu/droyster/courses/spring 04/index.html

[2] Sigl, R.: Ebene und Sphärische Trigonometrie. H. Wichmann-Verlag, Karlsruhe 1977.

[3] van der Waerden, B.L.: Polygone mit maximalem Flächeninhalt. Elem. Math. 5 (1950), 121-144.

Lienhard Wimmer

Dall' Armistr. 3

D-80638 München, Germany

e-mail: mathematics@lienhard-wimmer.com 